Pacific Journal of Mathematic 


\title{
RINGS OVER WHICH CERTAIN MODULES ARE INJECTIVE
}

\author{
Ann K. Boyle and K. R. Goodearl
}

This paper is concerned with rings for which all modules in one of the following classes are injective: simple modules, quasi-injective modules, or proper cyclic modules. Such rings are known as $V$-rings, $Q I$-rings, and $P C I$-rings, respectively. First, some conditions are developed under which the properties of being a $V$-ring, $Q I$-ring, or $P C I$-ring are left-right symmetric. In the next section, it is shown that a semiprime Goldie ring is a $Q I$-ring if and only if all singular quasi-injective modules are injective. An example is constructed to show that the class of $Q I$-rings is properly contained in the class of noetherian $V$-rings. Also, it is shown that the global homological dimension of a $Q I$-ring cannot be any larger than its Krull dimension. In the final section, it is shown that a $V$-ring is noetherian if and only if it has a Krull dimension. Examples are put forward to show that a noetherian $V$-ring may have arbitrary finite Krull dimension.

1. Introduction and definitions. A ring $R$ is said to be a right $V$-ring provided all simple right $R$-modules are injective. According to Villamayor [16, Theorem 2.1], this is equivalent to the condition that every right ideal of $R$ is an intersection of maximal right ideals. In particular, the Jacobson radical of any right $V$-ring is zero, and consequently all right $V$-rings are semiprime. For further properties and examples of $V$-rings, we refer the reader to Boyle [1], Cozzens [5], Cozzens-Johnson [6], Farkas-Snider [9], Michler-Villamayor [16], and Osofsky [17].

We recall that a module $A$ is quasi-injective provided every homomorphism from a submodule of $A$ into $A$ extends to an endomorphism of $A$. According to Johnson-Wong [13, Theorem 1.1], this is equivalent to the condition that $A$ be a fully invariant submodule of its injective hull, which we denote by $E(A)$. For example, any semisimple module (i.e., a module which is a sum of simple submodules) is quasi-injective. A ring $R$ is a right $Q I$-ring provided all quasiinjective right $R$-modules are injective. Inasmuch as all simple right $R$-modules are quasi-injective, it follows that $R$ must also be a right $V$-ring. In addition, since all semisimple right $R$-modules are quasiinjective and thus injective, we see from Kurshan [15, Theorem 2.4] that $R$ is right noetherian. Therefore: every right $Q I$-ring is a right 
noetherian, right $V$-ring. For further properties and examples of $Q I$-rings, we refer the reader to Boyle [1] and Byrd [2,3].

The proper cyclic right modules over a ring $R$ are those cyclic right $R$-modules $R / I$ for which $R / I \not R R$. We say that $R$ is a right PCI-ring provided all proper cyclic right $R$-modules are injective. If $R$ is not a division ring, then all simple $R$-modules are proper cyclic, from which we conclude that every right $P C I$-ring is also a right $V$ ring. According to Faith [7, Theorems 14, 17], a right $P C I$-ring is either semisimple or else is a simple, right semihereditary, right Ore domain. In view of this result, we shall only consider right PCIdomains in this paper.

The reader is assumed to be familiar with the notions of singular and nonsingular modules, as in [10], for example. Also we shall need the notions of Krull dimension and critical modules as developed in [11]. We remind the reader that a uniform module is one in which the intersection of any two nonzero submodules is nonzero. Finally, we use the notation $\operatorname{soc}(A)$ for the socle of a module, and r.gl.dim. $(R)$ for the right global dimension of a ring $R$.

2. Left-right symmetry. In this section we consider conditions under which a right $V$-ring ( $Q I$-ring, $P C I$-domain) is also a left $V$-ring ( $Q I$-ring, $P C I$-domain). These results are consequences of the existence of a duality (i.e., a contravariant category equivalence) between the categories of finitely generated singular right modules and finitely generated singular left modules. We note that in general a right $V$-ring need not be a left $V$-ring, as shown by an example of Michler and Villamayor [16, Remark 4.5]. For $Q I$-rings and PCI-domains, however, the question of left-right symmetry in general remains open.

LEMMA 1. Let $R$ be a right and left noetherian semiprime ring with classical quotient ring $Q$, and let $\mathscr{F}_{R}\left({ }_{R} \mathscr{F}\right)$ denote the category of all finitely generated singular right (left) $R$-modules. If $(Q / R)_{R}$ and ${ }_{R}(Q / R)$ are both injective, then there exists a duality

$$
\operatorname{Ext}_{R}^{1}(-, R): \mathscr{F}_{R} \rightarrow_{R} \mathscr{F}
$$

Proof. Inasmuch as $Q_{R}$ is nonsingular and injective, we observe that $\operatorname{Hom}_{R}\left(-,(Q / R)_{R}\right)$ and $\operatorname{Ext}_{R}^{1}\left(-, R_{R}\right)$ are naturally equivalent on $\mathscr{F}_{R}$. Using this, it follows readily that $\operatorname{Ext}_{R}^{1}\left(-, R_{R}\right)$ and $\operatorname{Ext}_{R}^{1}\left(-,{ }_{R} R\right)$ define contravariant functors $\mathscr{F}_{R} \rightarrow{ }_{R} \mathscr{F}$ and ${ }_{R} \mathscr{F} \rightarrow \mathscr{F}_{R}$ respectively, and all that remains is to show that both compositions of these two functors are naturally equivalent to the appropriate identity functors. 
In view of the above remarks, $\operatorname{Ext}_{R}^{1}\left(\operatorname{Ext}_{R}^{1}\left(-, R_{R}\right),{ }_{R} R\right)$ is naturally equivalent to $\operatorname{Hom}_{R}\left(\operatorname{Ext}_{R}^{1}\left(-, R_{R}\right),{ }_{R}(Q / R)\right)$ on $\mathscr{F}_{R}$. Since ${ }_{R}(Q / R)$ is injective by hypothesis, it follows from Cartan-Eilenberg [4, Proposition 5.3, p. 120] that $\operatorname{Hom}_{R}\left(\operatorname{Ext}_{R}^{1}\left(-, R_{R}\right),{ }_{R}(Q / R)\right)$ is naturally equivalent to $\operatorname{Tor}_{1}^{R}\left(-, \operatorname{Hom}_{R}\left({ }_{R} R,{ }_{R}(Q / R)\right)\right)$ on $\mathscr{F}_{R}$. Finally, we see by Sandomierski [18, Corollary, p. 119] that $\operatorname{Tor}_{1}^{R}(-, Q / R)$ is naturally equivalent to the identity functor on $\mathscr{F}_{R}$.

Proposition 2. Let $R$ be a right and left noetherian ring with maximal right quotient ring $Q$, and assume that $R$ is a right $V$-ring. If $(Q / R)_{R}$ and ${ }_{R}(Q / R)$ are both injective, then all singular right and left $R$-modules are semisimple and injective.

Proof. Since $R$ is a semiprime ring, $Q$ is the classical quotient ring of $R$. We thus obtain the duality between $\mathscr{F}_{R}$ and ${ }_{R} \mathscr{F}$ as in Lemma 1. Now $\mathscr{F}_{R}$ and $\mathscr{F}_{R} \mathscr{F}$ are noetherian categories, hence it follows from the duality that they are also artinian categories. Thus every object in each of these categories has a composition series. Since $R$ is a right $V$-ring, we infer from this that every object in $\mathscr{F}_{R}$ is semisimple, and then it follows from the duality that the same is true in ${ }_{R} \mathscr{F}$. In particular, $R / I$ must be semisimple for any essential right or left ideal $I$ of $R$. The proposition now follows from Goodearl [10, Proposition 3.1].

THEOREM 3. Suppose $R$ is a right and left noetherian ring with maximal right quotient ring. $Q$, and assume that $R$ is a right $V$ ring. Then $R$ is right and left hereditary if and only if $(Q / R)_{R}$ and ${ }_{R}(Q / R)$ are both injective.

Proof. As in Proposition 2, we see that $R$ is semiprime and that $Q$ is the classical quotient ring of $R$. Thus $Q_{R}$ and ${ }_{R} Q$ are both injective, hence if $R$ is right and left hereditary we automatically obtain $(Q / R)_{R}$ and ${ }_{R}(Q / R)$ injective. Conversely, if $Q / R$ is injective on both sides, Proposition 2 shows that all singular right and left $R$-modules are injective. According to Goodearl [10, Proposition 3.3], $R$ is thus right and left hereditary.

Corollary 4. Suppose that $R$ is a right and left QI-ring with maximal right quotient ring $Q$. Then $R$ is right and left hereditary if and only if $(Q / R)_{R}$ and ${ }_{R}(Q / R)$ are both injective.

Proof. Inasmuch as $R$ is a right and left noetherian, right and left $V$-ring, this is a direct consequence of Theorem 3. 
THEOREM 5. Let $R$ be a right and left noetherian ring with maximal right quotient ring $Q$, and assume that $(Q / R)_{R}$ and ${ }_{R}(Q / R)$ are both injective. Then the following conditions are equivalent:
(a) $R$ is a right $V$-ring.
(b) $R$ is a left $V$-ring.
(c) $R$ is a right QI-ring.
(d) $R$ is a left QI-ring.

Proof. Under any of the assumptions a,b, c, d, $R$ is a $V$-ring on one side or the other and hence is semiprime. Thus we may as well assume that $R$ is semiprime to begin with. In this case $Q$ is also the maximal left quotient ring of $R$, and so our hypotheses are now left-right symmetric.

$\mathrm{a} \Rightarrow \mathrm{b}$ : According to Faith [8, Theorem 31], $R$ is a finite direct product of simple rings, hence we need only consider the case when $R$ itself is simple. If $\operatorname{soc}\left({ }_{R} R\right) \neq 0$, then $\operatorname{soc}\left({ }_{R} R\right)=R$ and $R$ is a semisimple ring, whence (b) is automatic. On the other hand, if $\operatorname{soc}\left({ }_{R} R\right)=0$, then all simple left $R$-modules are singular, hence it follows from Proposition 2 that $R$ is a left $V$-ring.

$\mathrm{b} \Rightarrow \mathrm{a}:$ By symmetry.

$\mathrm{a} \Rightarrow \mathrm{c}$ : Inasmuch as $R$ is right and left hereditary by Theorem 3, this follows from Boyle [1, Theorem 5].

$c \Rightarrow a$ : is automatic, and then $b \Leftrightarrow d$ by symmetry.

COROLlaRy 6. Let $R$ be a right and left noetherian domain. Then $R$ is a right PCI-domain if and only if $R$ is a left PCI-domain.

Proof. If $R$ is a right noetherian PCI-domain, then according to Boyle [1, Theorem 7] $R$ is right hereditary. By Small [19, Corollary 3], $R$ is also left semihereditary and thus left hereditary. Letting $Q$ denote the classical quotient ring of $R$, we thus see that $(Q / R)_{R}$ and ${ }_{R}(Q / R)$ are both injective. Since $R$ is in particular a right $V$-ring, Theorem 5 now says that $R$ is also a left $V$-ring. Thus Boyle [1, Corollary 10] shows that $R$ is a left PCI-domain.

3. $Q I$-rings. This section is concerned with several aspects of the structure of $Q I$-rings. We begin by looking at semiprime Goldie rings, in which case we show that $R$ is a $Q I$-ring provided only that its singular quasi-injective modules are injective. Second, we give an example to show that not all noetherian $V$-rings need be $Q I$ rings. Finally, we prove several results about the structure of modules over a $Q I$-ring $R$ which lead to the inequality r.gl.dim. $R \leqq \mathrm{~K} . \operatorname{dim} . R$. 
LeмmA 7. Let $R$ be any right nonsingular ring. If $R_{R}$ is finitedimensional, then all faithful nonsingular quasi-injective right $R$ modules are injective.

Proof. Let $A$ be any faithful nonsingular quasi-injective right $R$-module. Since $A$ is nonsingular, the annihilator of any subset of $A$ is an $\mathscr{S}$-closed right ideal of $R$ in the sense of [10, p. 14]. According to Goddearl [10, Theorem 1.24], the finite-dimensionality of $R_{R}$ implies that the $\mathscr{S}$-closed right ideals of $R$ satisfy the descending chain condition. Thus $A$ must have a finite subset $\left\{a_{1}, \cdots, a_{n}\right\}$ whose annihilator is minimal among the annihilators of finite subsets of $A$, and then we infer from the faithfulness of $A$ that the annihilator of $\left\{a_{1}, \cdots, a_{n}\right\}$ is 0 . Consequently the element $\left(a_{1}, \cdots, a_{n}\right) \in A^{n}$ has zero annihilator, whence $A^{n}$ contains an isomorphic copy of $R_{R}$. Since $A^{n}$ is quasi-injective by Harada [12, Proposition 2.4], it follows easily from Baer's criterion that $A^{n}$ must be injective. Therefore $A$ is injective.

Unforunately, the hypothesis of faithfulness cannot be omitted from Lemma 7. For if $R$ is the ring of all lower triangular $2 \times 2$ matrices over a field $F$, then its radical $J$ is a minimal right ideal of $R$ and thus is a nonsingular quasi-injective right $R$-module. However, since $J$ contains no nonzero idempotents it cannot be injective.

Over a commutative noetherian nonsingular ring, faithfulness can be dropped, as shown by Harada [12, Propositions 2.5, 2.6]. This result carries over to semiprime Goldie rings, as the following theorem shows.

THEOREM 8. If $R$ is a semiprime right Goldie ring, then all nonsingular quasi-injective right $R$-modules are injective.

Proof. Let $A$ be any nonsingular quasi-injective right $R$-module, and let $Q$ denote the classical right quotient ring of $R$, which is a semisimple ring. Now $E(A)$ is nonsingular and so is a right $Q$-module, hence there exists a ring decomposition $Q=Q_{1} \oplus Q_{2}$ such that $E(A) Q_{1}=0$ and $E(A)$ is faithful over $Q_{2}$. If $I=R \cap Q_{1}$, then $I$ is a two-sided ideal of $R$ such that $E(A) I=0$. Since it suffices to show that $A$ is a direct summand of $E(A)$, it follows that we need only prove that $A$ is injective as an $(R / I)$-module. Inasmuch as $R / I$ is a semiprime right Goldie ring with classical right quotient ring $Q_{2}$, we may thus assume, without loss of generality, that $E(A)$ is a faithful right $Q$-module.

According to Lemma 7, it is enough to show that the annihilator $H=\{r \in R \mid A r=0\}$ is 0 . Since $E(A)$ is a faithful right module over the semisimple ring $Q$, it must contain a finite subset $\left\{x_{1}, \cdots, x_{n}\right\}$ whose 
annihilator in $Q$ is zero. There must be an essential right ideal $J$ of $R$ such that $x_{l} J \leqq A$ for all $i$, whence $x_{i} J H=0$ for all $i$, and thus $J H=0$. Now $H J$ is a nilpotent two-sided ideal of $R$, hence our semiprime hypothesis implies that $H J=0$. Inasmuch as $J$ is essential in $R$, we conclude that $H=0$, and so $A$ is indeed faithful.

COROllary 9. Let $R$ be a semiprime right Goldie ring. Then $R$ is a right $Q I$-ring if and only if all singular quasi-injective right $R$-modules are injective.

Proof. Assume that all singular quasi-injective right $R$-modules are injective, and consider an arbitrary quasi-injective right $R$-module $A$. The singular submodule $Z(A)$ is a fully invariant submodule of $A$ and thus is quasi-injective, hence we obtain that $Z(A)$ is injective. Now $A \cong Z(A) \oplus[A / Z(A)]$ and so $A / Z(A)$ is quasiinjective, whence Theorem 8 says that $A / Z(A)$ must be injective. Therefore $A$ is injective.

As we have remarked above, every $Q I$-ring is a noetherian $V$-ring, and Byrd [2] has raised the converse question of whether every noetherian $V$-ring must be a $Q I$-ring. The answer is no, as we now show.

EXAmPLE. There exists a right and left noetherian, right and left $V$-ring $R$ which is not a right $Q I$-ring.

Proof. Let $F$ be a universal differential field of characteristic 0 with respect to two commuting derivations $\delta_{1}$ and $\delta_{2}$ (Kolchin [14, Theorem, p. 771]), and let $R=F\left[\theta_{1}, \theta_{2}\right]$ be the ring of linear differential operators over $F$. We recall that the elements of $R$ are noncommutative polynomials in the indeterminates $\theta_{1}, \theta_{2}$, subject to the relations $\theta_{1} \theta_{2}=\theta_{2} \theta_{1}$ and $\theta_{i} \alpha=\alpha \theta_{1}+\delta_{i} \alpha$ for all $\alpha \in F$. It is easily seen that $R$ is a right and left noetherian ring. Cozzens and Johnson have shown in [6, Theorem 1] that $R$ is a left $V$-ring, and the same argument shows that $R$ is also a right $V$-ring.

In view of the relation $\theta_{i} \alpha=\alpha \theta_{l}+\delta_{l} \alpha$, we can extend $\delta_{i}$ to a derivation of $R$ by setting $\delta_{i} r=\theta_{i} r-r \theta_{i}$. Note that $R=F\left[\theta_{1}\right]\left[\theta_{2}\right]$, i.e., $R$ is equal to the ring of linear differential operators over the differential ring $\left(F\left[\theta_{1}\right], \delta_{2}\right)$. Likewise, $R=F\left[\theta_{2}\right]\left[\theta_{1}\right]$.

Inasmuch as $R=F\left[\theta_{1}\right]\left[\theta_{2}\right]=F\left[\theta_{1}\right]+\theta_{2} R$, we have $R / \theta_{2} R \cong F\left[\theta_{1}\right]$ as right $F\left[\theta_{1}\right]$-modules. We compute that the right $R$-module action on $F\left[\theta_{1}\right]$ is given by its right $F\left[\theta_{1}\right]$-module action together with the rule $x * \theta_{2}=-\delta_{2} x$. Now $F\left[\theta_{1}\right]$ is a right and left noetherian domain, hence 
it has a classical quotient division ring $Q$. We extend $\delta_{2}$ to a derivation of $Q$ according to the rule $\delta_{2}\left(a b^{-1}\right)=\left(\delta_{2} a\right) b^{-1}-a b^{-1}\left(\delta_{2} b\right) b^{-1}$, and then we make $Q$ into a right $R$-module by using its right $R$-module action together with the rule $x * \theta_{2}=-\delta_{2} x$.

Now $Q$ is uniform as a right $F\left[\theta_{1}\right]$-module and thus also as a right $R$-module. Therefore $E\left(Q_{R}\right)$ is an indecomposable right $R$ module. Considering $E\left(Q_{R}\right)$ just as a module over the noetherian domain $F\left[\theta_{2}\right]$ (which is a subring of $R$ ), it has a torsion submodule which we shall denote by $T$. We proceed by showing that $T$ is a right $R$-module which is quasi-injective but not injective. Since $T$ is an $F\left[\theta_{2}\right]$-submodule of $E\left(Q_{R}\right)$, and since $R=F\left[\theta_{2}\right]\left[\theta_{1}\right], T$ will be an $R$-submodule of $E\left(Q_{R}\right)$ provided $T \theta_{1} \subseteq T$. Given any $t \in T$, we have $t a=0$ for some nonzero $a \in F\left[\theta_{2}\right]$. Observing that $\delta_{1} a \in F\left[\theta_{2}\right]$, we compute that $t \theta_{1} a=t\left(\delta_{1} a\right) \in T$, and thus $t \theta_{1} \in T$. Therefore $T$ is indeed an $R$-submodule of $E\left(Q_{R}\right)$.

Inasmuch as $T$ is a fully invariant submodule of $E\left(Q_{R}\right), T$ is a quasi-injective right $R$-module. Observing that $1 * \theta_{2}=-\delta_{2} 1=0$, we see that $1 \in T$ and thus $T \neq 0$. Since $F$ is a universal differential field, there must be an element $\alpha \in F$ such that $\delta_{2} \alpha=1$. Then $\delta_{2}\left(\alpha+\theta_{1}\right)=1$ also, from which we compute that $\delta_{2}^{n}\left(\alpha+\theta_{1}\right)^{-1}=(-1)^{n} n !\left(\alpha+\theta_{1}\right)^{-1,-1}$ for all $n>0$. We now infer that the elements $\left(\alpha+\theta_{1}\right)^{-1} * \theta_{2}^{n}$ in $Q$ are right linearly independent over $F$, i.e., $\left(\alpha+\theta_{1}\right)^{-1}$ is not annihilated by any nonzero elements of $F\left[\theta_{2}\right]$. Thus $\left(\alpha+\theta_{1}\right)^{-1} \notin T$, and so $T \neq E\left(Q_{R}\right)$.

Now $E\left(Q_{R}\right)$ is indecomposable and $T$ is a nontrivial submodule of $E\left(Q_{R}\right)$, hence $T$ cannot be injective. Since $T$ is quasi-injective, $R$ cannot be a right $Q I$-ring.

Proposition 10. [8, Proposition 32]. Let $R$ be a right QI-ring. If $E$ is any nonzero indecomposable injective right $R$-module, then $\Lambda=$ $\operatorname{Hom}_{R}(E, E)$ is a division ring.

TheOREM 11. Let $R$ be a right QI-ring. If $A$ is any nonzero finitely generated right $R$-module with Krull dimension $\alpha$, then all finitely generated submodules of $E(A) / A$ have Krull dimension strictly less than $\alpha$.

Proof. First consider the case where $A$ is critical, and suppose $E(A) / A$ has a finitely generated submodule $B / A$ with $\mathrm{K} \cdot \operatorname{dim} .(B / A) \geqq$ $\alpha$. Since $R$ is a right noetherian, there exist submodules $B_{0}=A<B_{1}<$ $\cdots<B_{n}=B$ such that each $B_{k} / B_{k-1}$ is critical. We must have K.dim. $\left(B_{k} / B_{k-1}\right) \geqq \alpha$ for some $k$. Thus, replacing $B$ by $B_{k}$ and setting $C=B_{k-r}$, we have modules $A \leqq C<B$ such that $B / C$ is $\beta$-critical for some $\beta \geqq \alpha$. 
Now let $Q$ be the sum of all submodules $P$ of $E(A)$ for which $\operatorname{Hom}_{R}(P, E(B / C))=0$. We observe that $Q$ is a fully invariant submodule of $E(A)$, i.e., $Q$ is quasi-injective. Since $R$ is a right $Q I$-ring, $Q$ must be injective. Since $A$ is critical it must be uniform, and thus $E(A)$ is indecomposable, so the only choices for $Q$ are $E(A)$ or 0 . Inasmuch as the identity map on $B / C$ extends to a nonzero map $E(A) \rightarrow E(A) / C \rightarrow E(B / C)$, we obtain $Q \neq E(A)$ and thus $Q=0$.

Since $Q=0$, there exists a nonzero map $f: A \rightarrow E(B / C)$, which induces a nonzero map from $A_{0}=f^{-1}(B / C)$ into $B / C$. According to Gordon-Robson [11, Proposition 2.3], $A_{0}$ is $\alpha$-critical and all nonzero submodules of $B / C$ are $\beta$-critical. Inasmuch as $\beta \geqq \alpha$, it follows that $f$ must be a monomorphism, whence $A_{0}$ is isomorphic to a nonzero submodule $B_{0} / C$ of $B / C$. The map $B_{0} \rightarrow B_{0} / C \stackrel{\cong}{\rightarrow} A_{0}$ extends to a nonzero endomorphism $g$ of $E(A)$, and since $g A=0$ we see that $g$ cannot be an isomorphism. But then the endomorphism ring of $E(A)$ is not a division ring, which contradicts Proposition 10.

Thus the theorem holds for critical modules. In general, $A$ must have an essential submodule $K=K_{1} \oplus \cdots \oplus K_{n}$, where each $K_{i}$ is $\alpha_{i}$-critical for some $\alpha_{i} \leqq \alpha$. In view of the results above, every finitely generated submodule of $E\left(K_{i}\right) / K_{i}$ has Krull dimension strictly less than $\alpha_{i}$. It follows that every finitely generated submodule of $E(K) / K$ has Krull dimension strictly less than $\alpha$, from which the theorem follows.

COROllary 12. If $R$ is any right QI-ring, then r.gl.dim. $R \leqq$ K.dim.R.

Proof. We need only consider the case when K.dim.R $=N<$ $\infty$. For any right $R$-module $A$, let $\phi(A)$ denote the supremum of the Krull dimensions of all finitely generated submodules of $A$. Note that $\phi(A) \leqq N$, and that $\phi(A)=\mathrm{K} \cdot \operatorname{dim} . A$ when $A$ is finitely generated. It suffices to show that $i d_{R}(A) \leqq N$ for all nonzero right $R$-modules $A$, where $i d_{R}(A)$ denotes the injective dimension of $A$. We proceed by induction to show that $i d_{R}(A) \leqq \phi(A)$ for all nonzero $A$.

If $\phi(A)=0$, then since $R$ is right noetherian all finitely generated submodules of $A$ must have composition series. Inasmuch as $R$ is a right $V$-ring, all such submodules of $R$ must be semisimple, and thus $A$ itself is semisimple. Now $A$ is quasi-injective and therefore injective, whence $i d_{R}(A)=\phi(A)$.

Now let $\phi(A)=n>0$ and assume that $i d_{R}(B) \leqq \phi(B)$ for all nonzero modules $B$ with $\phi(B)<n$. Choose an essential submodule of $A$ of the form $K=\oplus K_{\alpha}$, where each $K_{\alpha}$ is finitely generated. According to Theorem 11, all finitely generated submodules of 
$E\left(K_{\alpha}\right) / K_{\alpha}$ have Krull dimension strictly less than K.dim. $K_{\alpha}$, from which we infer that $\phi\left(E\left(K_{\alpha}\right) / K_{\alpha}\right)<n$. It now follows that $\phi(E(A) / A)<n$, and then $i d_{R}(E(A) / A)<n$ by the induction hypothesis. Therefore $i d_{R}(A) \leqq n$, and the induction works.

4. $\quad V$-rings. In this section we consider the Krull dimension of $V$-rings. According to Michler-Villamayor [16, Theorem 4.2], a right $V$-ring with right Krull dimension at most one is right noetherian, right hereditary, and Morita-equivalent to a finite direct sum of simple $V$-domains. In general, all positive integers are possible as Krull dimensions of $V$-rings, and we exhibit examples of this. We also show that a $V$-ring has a Krull dimension if and only if it is already noetherian.

PROPOSITION 13. Let $R$ be a right $V$-ring. Then $R$ has right Krull dimension if and only if $R$ is right noetherian.

Proof. All right noetherian rings have right Krull dimension: Gordon-Robson [11, Proposition 1.3]. On the other hand, if $R$ is not right noetherian then by using an argument of Faith in [7, Corollary 15B] we infer that there exists a cyclic right $R$-module $E$ whose socle is an infinite direct sum of simple modules. But then $E$ is not finitedimensional and so does not have Krull dimension, by Gordon-Robson [11, Proposition 1.4]. Since $E$ is cyclic, it follows that $R$ does not have Krull dimension.

Corollary 14. If $R$ is a right PCI-domain, then the following conditions are equivalent:

(a) $R$ is right noetherian.

(b) $R$ has Krull dimension.

(c) $R$ has Krull dimension at most 1 .

Proof. Since $R$ is a right $V$-ring, a $\Leftrightarrow$ b by Proposition 13. If (a) holds, then $R$ is a simple, right hereditary ring by Faith [7, Theorem 14]. Inasmuch as $R$ is a right $P C I$-domain, we see that all cyclic submodules of any singular right $R$-module $A$ are direct summands of $A$. In case $A$ is finitely generated as well, then it must be finitedimensional since $R$ is right noetherian, and Goodearl [10, Proposition 1.22] shows that $A$ is semisimple. In particular, $R / I$ is semisimple for all essential right ideals $I$ of $R$, whence Goodearl [10, Proposition 3.1] shows that all singular right $R$-modules are semisimple and injective. It now follows from Michler-Villamayor [16, Theorem 4.2] that K.dim. $R \leqq 1$. 
It is an open question whether all right $P C I$-rings are right noetherian. Corollary 14 might provide a means for attacking this question.

Cozzens and Johnson [6] have constructed examples of noetherian $V$-rings with arbitrary finite global dimension, and we shall show that these examples also have arbitrary finite Krull dimension. Since these examples are differential operator rings, we begin with two general results on the Krull dimension of differential operator rings.

LEMMA 15. Let $R$ be any right noetherian ring with a derivation $\delta$. Then the ring of linear differential operators $R[\theta]$ is right noetherian, and

$$
\mathrm{K} \cdot \operatorname{dim} \cdot R[\theta] \leqq \mathrm{K} \cdot \operatorname{dim} \cdot R+1
$$

Proof. Given any right ideal $J$ of $R[\theta]$ and any nonnegative integer $n$, let $J_{n}$ be the set of all leading coefficients of elements of $J$ of degree $n$, together with 0 . Then the collection $\left\{J_{0}, J_{1}, \cdots\right\}$ is an ascending sequence of right ideals of $R$, hence we can define a right ideal in the ordinary polynomial ring $R[x]$ by setting $J_{0}+J_{1} x+J_{2} x^{2}+\cdots$. We thus obtain a monotone map $\phi$ from the right ideal lattice of $R[\theta]$ into the right ideal lattice of $R[x]$, and as in the Hilbert Basis Theorem an easy induction on degrees shows that $\phi$ is a strictly monotone map. Now $R[x]$ is certainly right noetherian, and K.dim. $R[x]=\mathrm{K} \cdot \operatorname{dim} \cdot R+1$ by Gordon-Robson [11, Theorem 9.2], from which the lemma follows.

Proposition 16. If $F$ is a field with a finite collection $\delta_{1}, \cdots, \delta_{n}$ of commuting derivations, then the ring of linear differential operators $R=F\left[\theta_{1}, \cdots, \theta_{n}\right]$ has Krull dimension $n$.

Proof. By induction on Lemma 15, we see that $R$ is a right noetherian ring with K.dim. $R \leqq n$. Now set $J_{k}=\theta_{k} R+\cdots+\theta_{n} R$ for $k=1, \cdots, n$, and set $J_{n+1}=0$. We prove by induction on $k$ that $\mathrm{K} \cdot \operatorname{dim} \cdot R / J_{k} \geqq k-1$. Since $R / J_{1} \neq 0$, we automatically have $\mathrm{K} \cdot \operatorname{dim} \cdot R / J_{1} \geqq 0$. Now let $1 \leqq k \leqq n$ and assume that $\mathrm{K} \cdot \operatorname{dim} \cdot R / J_{k} \geqq$ $k-1$. Inasmuch as the $\theta_{i}$ all commute, we see that $\theta_{k}^{m} J_{k+1} \leqq J_{k+1}$ for all $m>0$. In fact, we compute that $\left\{r \in R \mid \theta_{k}^{m} r \in J_{k+1}\right\}=J_{k+1}$, from which it follows that

$$
\left\{r \in R \mid \theta_{k}^{m} r \in \theta_{k}^{m+1} R+J_{k+1}\right\}=\theta_{k} R+J_{k+1}=J_{k},
$$

i.e.,

$$
\left(\theta_{k}^{m} R+J_{k+1}\right) /\left(\theta_{k}^{m+1} R+J_{k+1}\right) \cong R / J_{k}
$$


Since K.dim. $R / J_{k} \geqq k-1$, it follows that $K \cdot \operatorname{dim} \cdot R / J_{k+1} \geqq k$. Thus the induction works, hence we obtain $\mathrm{K} \cdot \operatorname{dim} . R \geqq n$.

EXAMPLE. Given any positive integer $n$, there exists a right and left noetherian, right and left $V$-ring $R_{n}$ such that K.dim. $R_{n}=n$.

Proof. Let $F$ be a universal differential field with respect to $n$ commuting derivations $\delta_{1}, \cdots, \delta_{n}$ (Kolchin [14, Theorem, p. 771]), and let $R_{n}$ be the ring of linear differential operators $F\left[\theta_{1}, \cdots, \theta_{n}\right]$. Then $R_{n}$ is a noetherian $V$-ring by Cozzens-Johnson [6, Theorem 1], and K.dim. $R_{n}=n$ by Proposition 15 .

\section{REFERENCES}

1. Anne K. Boyle, Hereditary QI-rings, Trans. Amer. Math Soc., (to appear).

2. K. A. Byrd, When are quasi-injectives injective?, Canad. Math Bull., 15 (1972), 599-600.

3. _- Rings whose quasi-injective modules are injective, Proc. Amer. Math Soc., 33 (1972), 235-240.

4. H. Cartan and S. Eilenberg, Homological Algebra, Princeton (1956), Princeton University Press.

5. J. H. Cozzens, Homological properties of the ring of differential polynomials, Bull. Amer. Math. Soc., 76 (1970), 75-79.

6. J. H. Cozzens and J. Johnson, Some applications of differential algebra to ring theory, Proc. Amer. Math. Soc., 31 (1972), 354-356.

7. Carl Faith, When are proper cyclics injective?, Pacific J. Math., 45 (1973), 97-112.

8. - Modules finite over endomorphism ring, in Lectures on Rings and Modules, SpringerVerlav Lecture Notes No. 246 (1972), 145-189.

9. D. R. Farkas and R. L. Snider, Group algebras whose simple modules are injective, Trans. Amer. Math. Soc., (to appear).

10. K. R. Goodearl, Singular torsion and the splitting properties, Amer. Math Soc. Memoirs No. 124, (1972).

11. R. Gordon and J. C. Robson, Krull dimension, Amer. Math. Soc. Memoirs No. 133, (1973).

12. M. Harada, Note on quasi-injective modules, Osaka J. Math., 2 (1965), 351-356.

13. R. E. Johnson and E. T. Wong, Quasi-injective modules and irreducible rings, J. London Math. Soc., 36 (1961), 260-268.

14. E. R. Kolchin, Galois theory of differential fields, Amer. J. Math., 75 (1953), 753-824.

15. R. P. Kurshan, Rings whose cyclic modules have finitely generated socle, J. Algebra, 15 (1970), 376-386.

16. G. Michler and O. E. Villamayor, On rings whose simple modules are injective, J. Algebra, 25 (1973), 185-201.

17. B. L. Osofsky, On twisted polynomial rings, J. Algebra, 18 (1971), 597-607.

18. F. L. Sandomierski, Semisimple maximal quotient rings, Trans. Amer. Math. Soc., 128 (1967), 112-120.

19. L. W. Small, Semihereditary rings, Bull. Amer. Math. Soc., 73 (1967), 656-658.

Received April 30, 1974.

UNIVERSITY OF UTAH 



\section{CONTENTS}

Zvi Artstein and John A. Burns, Integration of compact set-valued functions

J. A. Beachy and W. D. Blair, Rings whose faithful left ideals are cofaithful

Mark Benard, Characters and Schur indices of the unitary reflection group $[321]^{3}$

H. L. Bentley and B. J. Taylor, Wallman rings ............................. 15

E. Berman, Matrix rings over polynomial identity rings II ............... 37

Simeon M. Berman, A new characterization of characteristic functions of absolutely continuous distributions ........................ 323

Monte B. Boisen, Jr. and Philip B. Sheldon, Pre-Prüfer rings ............ 331

A. K. Boyle and K. R. Goodearl, Rings over which certain modules are injective

J. L. Brenner, R. M. Crabwell and J. Riddell, Covering theorems for finite nonabelian simple groups. $V$

H. H. Brungs, Three questions on duo rings .................................... 345

Iracema M. Bund, Birnbaum-Orlicz spaces of functions on groups ....351

John D. Elwin and Donald R. Short, Branched immersions between 2-manifolds of higher topological type

J. K. Finch, The single valued extension property on a Banach space ................................................................................. 61

J. R. Fisher, A Goldie theorem for differentiably prime rings ............ 71

Eric M. Friedlander, Extension functions for rank 2, torsion free abelian groups ...................................................................... 371

J. Froemke and R. Quackenbusch, The spectrum of an equational class of groupoids

B. J. Gardner, Radicals of supplementary semilattice sums of

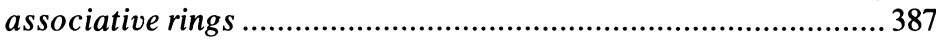

Shmuel Glasner, Relatively invariant measures ...............................393

G. R. Gordh, Jr. and Sibe Mardešić, Characterizing local connectedness in inverse limits...

S. Graf, On the existence of strong liftings in second countable topological spaces

S. Gudder and D. Strawther, Orthogonally additive and orthogonally increasing functions on vector spaces ........................................427

F. Hansen, On one-sided prime ideals .......................................... 79

D. J. Hartfiel and C. J. Maxson, A characterization of the maximal monoids and maximal groups in $\beta x$.

Robert E. Hartwig and S. Brent Morris, The universal flip matrix and the generalized faro-shuffle 


\section{Pacific Journal of Mathematics}

Vol. 58, No. 1

March, 1975

John Allen Beachy and William David Blair, Rings whose faithful left ideals are cofaithful .................................... 1

Herschel Lamar Bentley and Barbara June Taylor, Wallman rings ........ 15

Elizabeth Berman, Matrix rings over polynomial identity rings. II ...... 37

Ann K. Boyle and Kenneth R. Goodearl, Rings over which certain modules are injective ................................. 43

J. L. Brenner, Robert Myrl Cranwell and James Riddell, Covering theorems for finite nonabelian simple groups. $V \ldots \ldots \ldots \ldots \ldots \ldots \ldots \ldots \ldots$

James Kenneth Finch, The single valued extension property on a Banach

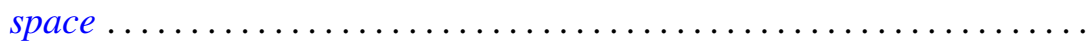

John Robert Fisher, A Goldie theorem for differentiably prime rings........ Friedhelm Hansen, On one-sided prime ideals .................... Jon Craig Helton, Product integrals and the solution of integral equations..........................................

Barry E. Johnson and James Patrick Williams, The range of a normal

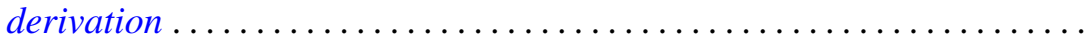

Kurt Kreith, A dynamical criterion for conjugate points ............ 123

Robert Allen McCoy, Baire spaces and hyperspaces .............. 133

John McDonald, Isometries of the disk algebra ................ 143

H. Minc, Doubly stochastic matrices with minimal permanents ......... 155

Shahbaz Noorvash, Covering the vertices of a graph by vertex-disjoint paths. ...

Theodore Windle Palmer, Jordan *-homomorphisms between reduced Banach*-algebras

Donald Steven Passman, On the semisimplicity of group rings of some

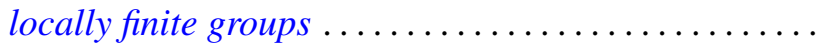

Mario Petrich, Varieties of orthodox bands of groups .

Robert Horace Redfield, The generalized interval topology on distributive



James Wilson Stepp, Algebraic maximal semilattices .... . .

Patrick Noble Stewart, A sheaf theoretic representation of rings with Boolean orthogonalities ........................

Ting-On To and Kai Wing Yip, A generalized Jensen's inequality......... 255

Arnold Lewis Villone, Second order differential operators with self-adjoint

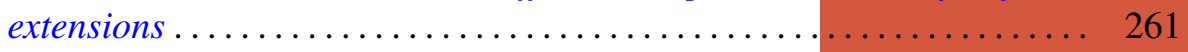

Martin E. Walter, On the structure of the Fourier-Stieltjes algebra ....... 267

John Wermer, Subharmonicity and hulls .................... 283

Edythe Parker Woodruff, A map of $E^{3}$ onto $E^{3}$ taking no disk onto a

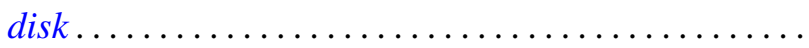

